\section{Condicionalidades de saúde no Programa Bolsa Família e a vigilância alimentar e nutricional: narrativas de profissionais da atenção primária à saúde}

\section{Health conditionalities in the Brazilian Income Transfer Program and food and nutritional surveillance: narratives by primary healthcare workers}

\section{Condicionalidades de salud en el Programa Bolsa Familia y vigilancia alimentaria y nutricional: relatos de profesionales de la atención primaria en salud}

Jorginete de Jesus Damião 1

Evelyne Lobato 1

Juliana Paulo e Silva 2

Claudia Valéria Cardim da Silva 1 Luciana Maria Cerqueira Castro 1 Luciana Azevedo Maldonado 1 Alexandro Alves Ribeiro 2

\section{Resumo}

Buscou-se compreender as narrativas dos profissionais da atenção primária à saúde do Município do Rio de Janeiro, Brasil, sobre os desafios e as potencialidades das ações de vigilância alimentar e nutricional, no contexto do Programa Bolsa Família, frente aos limites de sua implementação. Trata-se de pesquisa com abordagem qualitativa, avaliativa e participativa. Foram realizados, entre 2018 e 2019, cinco grupos focais (GF) com 60 profissionais de nivel superior de 24 unidades de saúde e um GF com 13 agentes comunitários de saúde. Com base no material transcrito dos GF foram construídas narrativas para cada grupo, produzindo uma síntese dos núcleos argumentais presentes nas falas. Posteriormente, foram realizados cinco grupos hermenêuticos para validação das narrativas e aprofundamento das discussões. No último encontro foram identificados os problemas e propostas relacionadas ao acompanhamento do Programa Bolsa Família. Na prática, o acompanhamento das condicionalidades é reduzido a "pesar e medir" as pessoas, sem que necessariamente se realize o diagnóstico nutricional para identificação de casos de risco e intervenção. Práticas como o "mutirão" no final da vigência do Programa Bolsa Família e a realização de medidas antropométricas pelos agentes comunitários de saúde, sem vinculação com a agenda das linhas de cuidado existentes, contribuem para este cenário. Prevalece uma visão burocrática dessas condicionalidades de saúde, apenas para garantir que a família não tenha o benefício cortado e o cumprimento das metas de gestão do Programa Bolsa Família. Ao longo da pesquisa, os profissionais construíram uma reflexão crítica desta prática e reconheceram sua potencialidade para o cuidado em saúde e nutrição das famílias.

Atenção Primária à Saúde; Política Nutricional; Vigilância Nutricional; Segurança Alimentar

\author{
Correspondência \\ J. J. Damião \\ Departamento de Nutrição Social, Instituto de Nutrição, \\ Universidade do Estado do Rio de Janeiro. \\ Rua São Francisco Xavier 524, 2 o andar, Bloco D, sala 12.007, \\ Rio de Janeiro, RJ 20559-900, Brasil. \\ jjdamiao@yahoo.com.br \\ 1 Universidade do Estado do Rio de Janeiro, Rio de Janeiro, \\ Brasil. \\ 2 Secretaria Municipal de Saúde do Rio de Janeiro, Rio de \\ Janeiro, Brasil.
}




\section{Introdução}

O Programa Bolsa Família conjuga a transferência direta de renda para as famílias com condicionalidades relacionadas às políticas de saúde e de Educação. Ele apresenta entre seus objetivos o combate à pobreza e a promoção do acesso à rede de serviços públicos, além do combate à fome e a promoção da segurança alimentar e nutricional 1.

O Programa Bolsa Família pode interferir no enfrentamento das situações de insegurança alimentar e nutricional, tanto na sua dimensão alimentar, proporcionando maior acesso a alimentos por meio da transferência de renda, quanto na dimensão nutricional, que diz respeito à relação com a saúde.

Diferentes estudos têm demonstrado impacto positivo para a segurança alimentar e nutricional das famílias acompanhadas, sobretudo em relação ao acesso à alimentação 2,3. A ampliação do acesso a direitos sociais básicos favorece a redução da vulnerabilidade social das famílias, impactando na situação de insegurança alimentar e nutricional, cujo enfrentamento requer uma abordagem intersetorial, de modo a incidir sobre seus múltiplos determinantes 2,3,4. No entanto, esse potencial das condicionalidades do Programa Bolsa Família depende da disponibilidade destes serviços, assim como da qualidade das ações ofertadas 5 .

No contexto do Sistema Único de Saúde (SUS), o acompanhamento das condicionalidades de saúde é um instrumento para a garantia da equidade, por estimular o acesso aos serviços de saúde de pessoas em situação de vulnerabilidade social. Os documentos oficiais sobre o Programa Bolsa Família enfatizam a oferta de serviços para a realização do pré-natal pelas gestantes, do acompanhamento do crescimento e desenvolvimento infantil e de imunização, ações que fazem parte das linhas de cuidado à saúde da mulher e da criança nos serviços da atenção primária à saúde (APS) 1 .

Contudo, a ênfase no monitoramento dessas ações acaba sendo traduzida no cotidiano dos serviços apenas para o cumprimento da condicionalidade em si, mas distante do seu papel na garantia do direito ao acesso e ao cuidado integral à saúde 6 .

O setor saúde tem um papel estratégico na agenda de segurança alimentar e nutricional, formalizado pelas ações previstas na Política Nacional de Alimentação e Nutrição (PNAN) 7, incluindo a organização da atenção nutricional e a vigilância alimentar e nutricional - esta última, um dos componentes do acompanhamento das condicionalidades de saúde do Programa Bolsa Família. Esta integração possibilita que a vigilância alimentar e nutricional do público infantil e de gestantes seja reforçada na agenda de compromissos das famílias e dos serviços de saúde, consequentemente promovendo o aumento da cobertura do acompanhamento do estado nutricional 1,6.

Desde a implementação das condicionalidades de saúde do Programa Bolsa Família, os profissionais de saúde demonstram resistência, que ocorrem mais por questões conceituais do que técnicas 8. Como são esses profissionais que implementam as políticas no cotidiano dos serviços de saúde, a escuta desses atores, com a produção e análise de suas narrativas, pode trazer elementos para compreender as potencialidades e os desafios dessas ações.

$\mathrm{O}$ estudo que subsidiou este artigo foi desenvolvido no âmbito da pesquisa intitulada Condicionalidades do Programa Bolsa Família: Estratégia para Garantir Equidade em Saúde e Nutrição. Neste artigo buscou-se compreender as narrativas dos profissionais de saúde da APS do Município do Rio de Janeiro, Brasil, acerca das ações referentes à vigilância alimentar e nutricional no contexto do Programa Bolsa Família.

\section{Métodos}

Trata-se de uma pesquisa com abordagem metodológica qualitativa, avaliativa e participativa, tomando como referência a proposta de Onocko Campos et al. ${ }^{9}$ para delineamento do desenho do estudo e construção e análise de narrativas.

As narrativas são histórias ainda não narradas 10 , nas quais objetos simbólicos, presentes na cultura, podem ser compartilhados a partir da interação com o público 9. Para Ricoeur 10, as narrativas constituem mediações entre o discurso e a ação. Assim, estas compõem uma síntese dos núcleos argu- 
mentais presentes nas falas, encadeados a partir do fluxo de ideias, fatos e afetos das histórias contadas e se conectam ao contexto social de vida 9 .

Sem negar a existência de sentidos latentes, ou seja, o significado por trás da fala dos sujeitos, esta proposta optou por construir um caminho de fala pela implicação e olhar avaliativo destes na construção do consenso 9,10,11.

\section{Critérios de seleção e perfil dos profissionais de saúde participantes}

As unidades de saúde foram selecionadas por amostragem propositiva, segundo características particulares em relação a critérios-chave 12 , conforme o Quadro 1.

O trabalho de campo foi pactuado com gestores da Secretaria Municipal de Saúde do Rio de Janeiro (SMS-RJ). Os responsáveis pelo Programa Bolsa Família e pelos Centros de Estudos de cada região de saúde mediaram o contato com os gestores das unidades de saúde selecionadas, que indicaram os profissionais para participação no estudo.

Cada unidade foi convidada a indicar até quatro profissionais de nível superior, conforme descrito no Quadro 1. Foi solicitada a indicação do dobro do número esperado de profissionais, para uma média de 10 a 15 participantes por grupo focal (GF), prevendo perdas devido à dificuldade de liberação destes.

A Tabela 1 apresenta o quantitativo de profissionais e unidades de saúde que participaram das três etapas da pesquisa. Participaram profissionais de 24 (66,7\%) unidades da APS, de nove das 10 regiões de saúde. Uma das regiões declinou, conforme a justificativa da gestão regional, em função da preocupação com a adesão dos profissionais que, pela "crise" na APS municipal, estavam sem pagamento dos salários. Cada GF reuniu profissionais de uma a três regiões de saúde, segundo proximidade territorial.

Inicialmente, não foi prevista a inclusão dos agentes comunitários de saúde (ACS) na estratégia metodológica, mas foi pactuada junto à gestão da SMS-RJ, a realização de um GF com 13 ACS de uma das unidades de saúde participantes, em função da expressiva participação no acompanhamento das condicionalidades do Programa Bolsa Família. A escolha por esta unidade se deu pela diversidade de território e modo de organização do cuidado, ambos sinalizados na fala dos profissionais nas etapas realizadas anteriormente.

A maioria dos profissionais do estudo era do sexo feminino (83,6\%), na faixa etária de 31 a 40 anos (38,3\%), de raça/cor negra (50,7\%). A síntese do perfil dos participantes é apresentada na Tabela 2.

\section{Quadro 1}

Critérios de seleção da amostra para os grupos focais.

\begin{tabular}{|c|c|c|}
\hline PARTICIPANTES & CRITÉRIOS DE SELEÇÃO & TOTAL DE PARTICIPANTES \\
\hline \multirow[t]{3}{*}{ Unidades de saúde } & Representações de todas as 10 regiões de saúde municipais & \multirow[t]{3}{*}{36 unidades } \\
\hline & $\begin{array}{l}\text { Equilíbrio entre unidades de atenção primária à saúde que não alcançaram } \\
\text { e que ultrapassaram a meta municipal de } 60 \% \text { de acompanhamento do } \\
\text { Programa Bolsa Família de cada região de saúde de acordo com dados } \\
\text { públicos referentes ao acompanhamento do Programa Bolsa Família da } \\
\text { segunda vigência de } 2017\end{array}$ & \\
\hline & $\begin{array}{l}\text { Equilíbrio entre unidades com e sem modelo } \\
\text { Estratégia Saúde da Família implantados }\end{array}$ & \\
\hline Profissionais de saúde & $\begin{array}{c}\text { Cada gerente de unidade de saúde foi convidado a indicar até } 4 \\
\text { profissionais: } 1 \text { médico; } 2 \text { enfermeiros e } 1 \text { profissional do Núcleo Ampliado } \\
\text { à Saúde da Família }\end{array}$ & $\begin{array}{c}\text { Até } 144 \text { participantes convidados, } \\
\text { prevendo uma perda de cerca de } 50 \%\end{array}$ \\
\hline
\end{tabular}

Fonte: elaborado pelos autores. 
Tabela 1

Participação por etapa da pesquisa.

\begin{tabular}{|c|c|c|c|c|c|c|}
\hline \multirow[t]{2}{*}{ Etapas da pesquisa } & \multicolumn{2}{|c|}{ Grupos focais * } & \multicolumn{2}{|c|}{ Grupos hermenêuticos * } & \multicolumn{2}{|c|}{ Encontro de avaliação e proposição ** } \\
\hline & $\begin{array}{c}\text { Convidados } \\
\text { n (\%) }\end{array}$ & $\begin{array}{c}\text { Participantes } \\
\text { n (\%) }\end{array}$ & $\begin{array}{c}\text { Convidados } \\
\text { n (\%) }\end{array}$ & $\begin{array}{c}\text { Participantes } \\
\text { n (\%) }\end{array}$ & $\begin{array}{c}\text { Convidados } \\
\text { n (\%) }\end{array}$ & $\begin{array}{c}\text { Participantes } \\
\text { n (\%) }\end{array}$ \\
\hline Unidades de saúde & $36(100,0)$ & $24(66,7)$ & $24(100,0)$ & $11(45,8)$ & $24(100,0)$ & $11(41,6)$ \\
\hline Profissionais de saúde & $144(100,0)$ & $60(41,7)$ & $60(100,0)$ & $24(40,0)$ & $62(100,0)$ & $19(31,6)$ \\
\hline
\end{tabular}

Fonte: elaborado pelos autores.

* Este número não inclui os agentes comunitários de saúde participantes, pois estes foram convidados posteriormente à realização dos grupos hermenêuticos;

** Dois agentes comunitários de saúde foram liberados pelo gestor e selecionados pelo próprio grupo para participação no encontro de avaliação e proposição.

Tabela 2

Perfil dos profissionais de saúde participantes da pesquisa.

\begin{tabular}{|c|c|c|}
\hline Características & $\mathrm{n}$ & $\%$ \\
\hline \multicolumn{3}{|l|}{ Idade (anos) } \\
\hline $26-30$ & 18 & 24,7 \\
\hline $31-40$ & 28 & 38,3 \\
\hline $41-50$ & 18 & 24,7 \\
\hline Acima de 50 & 7 & 9,6 \\
\hline Não informado & 2 & 2,7 \\
\hline \multicolumn{3}{|l|}{ Sexo } \\
\hline Feminino & 61 & 83,6 \\
\hline Masculino & 12 & 16,4 \\
\hline \multicolumn{3}{|l|}{ Raça/Cor } \\
\hline Amarela & 1 & 1,4 \\
\hline Branca & 32 & 43,8 \\
\hline Negra & 37 & 50,7 \\
\hline Não informado & 3 & 4,1 \\
\hline \multicolumn{3}{|l|}{ Categoria profissional } \\
\hline Agente comunitário de saúde & 13 & 17,8 \\
\hline Assistente social & 7 & 9,6 \\
\hline Educador físico & 6 & 8,2 \\
\hline Enfermeiro & 28 & 38,4 \\
\hline Fisioterapeuta & 2 & 2,7 \\
\hline Médico & 13 & 17,8 \\
\hline Nutricionista & 3 & 4,1 \\
\hline Psicólogo & 1 & 1,4 \\
\hline
\end{tabular}

Fonte: elaborado pelos autores.

Nota: estão incluídos nesta tabela os agentes comunitários de saúde, totalizando 73 profissionais de saúde participantes das etapas da pesquisa. 


\section{Etapas da pesquisa, organização e análise dos dados}

O estudo foi desenvolvido com profissionais de saúde da APS do Município do Rio de Janeiro, entre 2018 e 2019, e organizado em três etapas de trabalho de campo, que conformaram o caminho metodológico da pesquisa (Figura 1): GF, grupos hermenêuticos $(\mathrm{GH})$ e encontro de avaliação e proposição.

A primeira etapa consistiu na realização de GF que abordaram percepções e vivências dos atores envolvidos no acompanhamento das condicionalidades de saúde do Programa Bolsa Família. Estes foram conduzidos com base num roteiro pré-testado em unidade de saúde que não participou do estudo, contemplando as seguintes dimensões: (a) percepções dos profissionais quanto ao Programa Bolsa Família; (b) organização do acompanhamento; (c) conceito e papel das condicionalidades de saúde; (d) necessidades de saúde e de segurança alimentar e nutricional das famílias; (e) propostas de modificações e melhorias.

\section{Figura 1}

Fluxograma de descrição e objetivos do caminho metodológico.

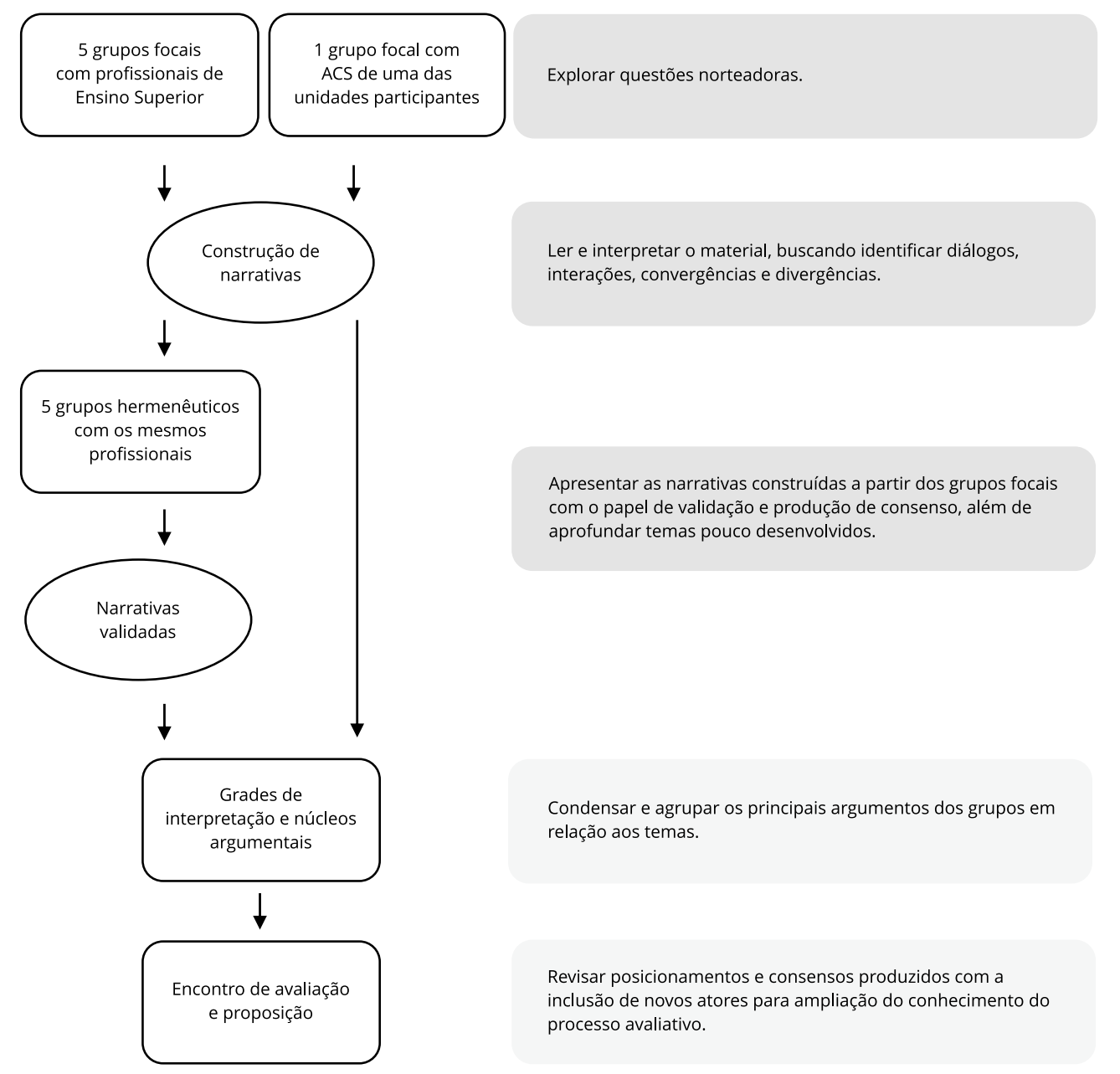

ACS: agentes comunitários de saúde.

Fonte: elaborado pelos autores. 
Os GF contaram com um moderador e dois relatores, integrantes da equipe de pesquisa. Foi utilizado o software QSR Nvivo versão 11 (https://www.qsrinternational.com/nvivo/home) para a organização do material transcrito dos GF segundo as dimensões do roteiro, em que foram identificados trechos que sintetizavam os núcleos argumentais, ou seja, os principais argumentos discutidos nos GF e utilizados para construção das narrativas referentes a cada grupo. As relatorias auxiliaram esta construção, seja no apoio à identificação dos núcleos argumentais ou no encadeamento lógico que procurou deixar evidentes as argumentações, consensos e divergências 9 .

Na segunda etapa do trabalho de campo, os mesmos profissionais que participaram dos GF foram convidados a participar dos GH. A dinâmica utilizada em cada GH consistiu na leitura da narrativa daquele grupo, a fim de validá-la e aprofundar algumas discussões. Este novo olhar para a narrativa oportunizou o reconhecimento das falas, o respeito aos dissensos, a atenuação de algumas críticas e a concretização da proposta metodológica participativa. O GF com os ACS foi realizado após esta etapa e não houve GH correspondente.

Após validação e ajustes nas narrativas foi realizada análise a partir de temáticas, sintetizadas em um quadro com questões e propostas/potências, apresentado no encontro de avaliação e proposição. Esta terceira e última etapa integrou a narrativa e participação dos ACS e buscou revisar posicionamentos e consensos produzidos para a ampliação do processo avaliativo. Constituiu ainda uma devolutiva dos resultados da pesquisa, que incluiu também gestores das regiões de saúde e do nível central da SMS-RJ.

O projeto de pesquisa foi financiado pelo Conselho Nacional de Desenvolvimento Científico e Tecnológico (CNPq; processo: 408235/2017-9) - e aprovado pelos Comitês de Ética em Pesquisa da Universidade do Estado do Rio de Janeiro (UERJ; CAAE no 84884118.5.0000.5259) e da SMS-RJ (CAAE no 84884118.5.3001.5279).

\section{Resultados e discussão}

Na agenda de acompanhamento das condicionalidades de saúde do Programa Bolsa família, proposta para as unidades da APS, são priorizadas as ações voltadas ao acompanhamento do pré-natal para gestantes, do crescimento das crianças e do calendário vacinal. Estas são as informações que o Governo Federal exige que os municípios informem a cada vigência semestral 1,13. Este registro no sistema de informação das condicionalidades de saúde do Programa Bolsa Família é realizado pelos profissionais da APS.

No processo de análise dos dados do estudo foram identificados temas e subtemas referentes à vigilância alimentar e nutricional das famílias acompanhadas, que estão resumidos no Quadro 2.

\section{A cultura do "pesar e medir"}

$\mathrm{Na}$ fala dos profissionais, o acompanhamento das condicionalidades de saúde é reduzido à ação de vigilância alimentar e nutricional, a qual é também reduzida à coleta e registro de peso e estatura, sobretudo das crianças das famílias atendidas pelo Programa Bolsa Família, como encontrado em outros estudos 8,14,15. Esta ação é identificada por eles como "pesar e medir" e sintetiza a percepção sobre como é ofertado este acompanhamento, distante de seu papel como estratégia para ampliar a equidade no acesso à saúde.

Esta "cultura do pesar e medir" foi construída desde a implantação do Programa Bolsa Família, com ênfase no registro das informações, desarticulada dos objetivos do programa e reproduzida por profissionais e famílias. Isso se expressa nas narrativas, que sinalizam que muitas famílias chegam com peso e estatura anotados para entregar ao profissional.

"As próprias beneficiárias estão acostumadas a chegar lá e jogar o peso. No início, tinha paciente que chegava com o papelzinho: 'Aqui, ó, a listagem do peso e altura dos meus filhos”'.

Os profissionais reclamam de certa resistência das famílias quando lhes é ofertada uma ação de cuidado em saúde para além do registro de peso e estatura, como consultas e grupos de educação em saúde. 


\section{Quadro 2}

Temas e síntese das ideias principais sobre a vigilância alimentar e nutricional no contexto do Programa Bolsa Família, a partir da narrativa dos profissionais de saúde. Rio de Janeiro, Brasil, 2018-2019.

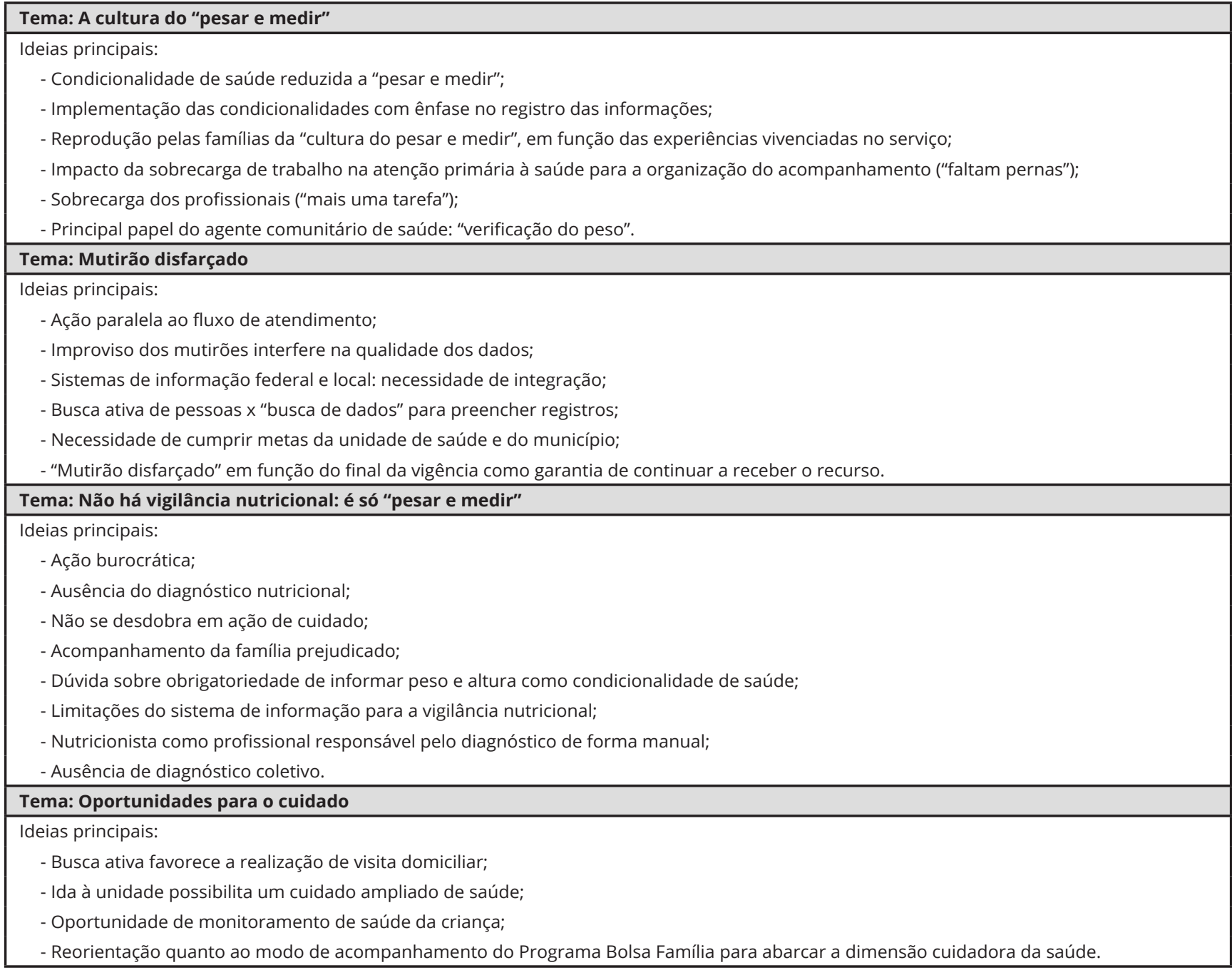

Fonte: elaborado pelos autores.

A dinâmica na oferta dos serviços de saúde modela as necessidades em demandas, que são explicitadas pela população 16. Uma vez que, na experiência das famílias no acompanhamento das condicionalidades de saúde, o "pesar e medir" é oferecido como a ação para garantir a permanência no programa, a necessidade por trás dessa demanda é a de continuar recebendo o Programa Bolsa Família.

Mas apesar de existir, por vezes, uma compreensão de que esta ação reduzida ao "pesar e medir", sem vínculo com outras ações de cuidado, não é suficiente, este continua sendo o modelo de acompanhamento mais frequente por diferentes motivos. A organização destas ações é afetada pelo julgamento dos profissionais de saúde em relação ao Programa Bolsa Família, como um programa assistencialista, que dá margem para irregularidades e não oferece mecanismos de saída das famílias; e em relação aos responsáveis das famílias, como que acomodados à situação de pobreza e isentos da corresponsabilidade com sua saúde e a de seus filhos. Da mesma forma, essa ação é influenciada pelas condições de trabalho dos profissionais na APS: "faltam pernas para a gente realizar um acompanhamento ideal". 
Após uma década de investimentos na reestruturação da APS, com a revisão da Política Nacional de Atenção Básica, assistimos a retrocessos no modelo assistencial da Estratégia Saúde da Família (ESF), tais como, a possibilidade de redução de ACS, desincentivos à abordagem territorial e comunitária com o novo modelo de financiamento da APS e a extinção do financiamento federal ao Núcleo Ampliado de Saúde da Família (NASF), entre outros, que representam ameaças, que somadas a problemas cronicamente enfrentados, ferem os princípios do SUS e impactam no cotidiano das equipes 17. O acompanhamento é "mais uma tarefa” para ocupar a equipe, que então realiza o que é possível.

"Se a equipe está sem médico, o enfermeiro tem que acolher a demanda dos pacientes das linhas de cuidado e ainda tem que acolher ao Bolsa Família. Acaba sendo assim: 'Quem vai pesar hoje?’.

Este contexto legitima a inclusão do ACS na realização da aferição do peso e estatura. Na rede de saúde do Município do Rio de Janeiro a avaliação antropométrica não é realizada pelo ACS, exceto para o acompanhamento das condicionalidades do Programa Bolsa Família e ações como o Programa Saúde na Escola. Documentos do Ministério da Saúde sobre as atribuições do ACS e sua inserção nas ações do Programa Bolsa Família referem à coleta de dados para a vigilância nutricional 18 e a verificação antropométrica 19 como sua atribuição, condicionada à supervisão de profissional de Nível Superior.

"Hoje, em algumas unidades, o agente comunitário tem que pesar. Será que é uma atribuição dele? É aquela questão do período de final da vigência. Para que o processo seja mais rápido, para dar conta em um determinado tempo, então solicitamos ao agente [ACS], que tem um número maior".

Os ACS realizam a aferição de peso e estatura e registram no sistema de informação, mas não realizam o diagnóstico nutricional, o que também não é realizado por outro profissional posteriormente.

"E muitas vezes não somos nós que alimentamos [o sistema de informação], porque temos que fazer outras coisas, atender a demanda que está lá fora, então a gente pega o ACS e fala: 'Vai lá: peso, altura e lança no sistema!"'.

\section{Mutirão disfarçado}

Desde a implementação do acompanhamento das condicionalidades o formato de "mutirão" é utilizado, seja na própria unidade de saúde, em paralelo ao fluxo dos atendimentos, seja em outros espaços do território, como equipamentos de assistência social e escolas. O improviso dos mutirões, muitas vezes, envolvia apenas a pesagem em locais e equipamentos inadequados, por profissionais pouco capacitados, comprometendo a qualidade dos dados e as ações de intervenção.

Nem todas as unidades continuam a realizar mutirões do Programa Bolsa Família. A integração do prontuário eletrônico local com o sistema federal do Programa Bolsa Família modificou a rotina do acompanhamento das condicionalidades entre 2015 e 2019 (em 2019 o município implantou o e-SUS - https://sisaps.saude.gov.br/esus/, sem interoperabilidade com o sistema do Programa Bolsa Família). Com esta integração, as informações registradas nos prontuários de atendimento na ESF migravam para o sistema federal. Faltando um mês para terminar a vigência, as equipes da ESF recebiam, da gestão municipal do Programa Bolsa Família, relatório das famílias que não passaram pelo acompanhamento de saúde.

"Tá chegando novembro, dezembro e tem 50 famílias sem acompanhamento. Aí é uma correria para busca ativa. Não o que a gente entende como busca ativa de um acompanhamento de Saúde da Família, isso vamos continuar fazendo. É aquela correria de buscar dado. Isso, na prática, realmente não funciona”.

Neste trecho da narrativa fica evidente que esta prática incomoda os profissionais, uma vez que não se trata de estratégia para subsidiar uma intervenção, mas para cumprir a meta de acompanhamento, ou seja, uma "busca de dados" daquelas famílias que não compareceram às consultas semestrais. Ação que não se assemelha à estratégia de "busca ativa”, tradicionalmente utilizada pela ESF 1.

A necessidade de cumprir a meta de acompanhamento do Programa Bolsa Família ao final da vigência leva à organização desta ação por meio de "mutirão disfarçado", no qual as pessoas são chamadas apenas para "pesar e medir", ou através de visitas domiciliares para o mesmo fim.

"Mas quando chega no final, a gente tem que fazer aquele mutirãozinho disfarçado. Busca um a um (...) Porque esse mutirão que não pode, é esse que é só pesar e medir".

O termo "mutirão disfarçado" aparece, pois em algumas regiões de saúde existe um entendimento de que a estratégia de mutirão, nos moldes que descrevemos anteriormente, não deve mais ser reali- 
zada. Mas, na fala dos profissionais, ao final da vigência, se justifica apenas "pesar e medir" visando informar o cumprimento das condicionalidades para que as pessoas não deixem de receber o recurso e para garantir as metas da unidade de saúde e do município em relação ao acompanhamento das condicionalidades. Uma visão burocrática do acompanhamento das condicionalidades de saúde.

\section{Não há vigilância nutricional: é só "pesar e medir"}

O diagnóstico nutricional é previsto em diferentes linhas de cuidado à saúde. Os profissionais reconhecem que monitorar o crescimento e o desenvolvimento é inerente ao acompanhamento integral da criança. No entanto, ainda que a prática de "pesar e medir" seja referenciada como prioritária para o acompanhamento das condicionalidades de saúde do Programa Bolsa Família, esta não necessariamente se desdobra em avaliação nutricional e, em consequência, não se concretiza em uma ação de cuidado de saúde, nem possibilita o monitoramento do estado nutricional deste grupo, como mostrado em outros estudos 6,8 .

"Individualmente, quando as medidas antropométricas são coletadas fora do momento da consulta, seja em visita domiciliar, seja em mutirões no território, seja no "mutirão disfarçado" na unidade, o diagnóstico nutricional não é realizado e, portanto, não se informa à pessoa avaliada ou à família sobre este".

É necessário compreender o contexto no qual a condicionalidade do "pesar e medir" foi construída. O estado nutricional era um dos critérios de seleção do antigo Programa Bolsa Alimentação, com foco no combate à desnutrição. Naquele momento de baixa cobertura da ESF, existia uma rotina de realização de pré-consultas pela equipe de enfermagem. Era um contexto diferente do atual na APS municipal, em que todas as aferições são realizadas no consultório pelo médico ou enfermeiro.

Mesmo entre os profissionais, não há certeza da obrigatoriedade de informar os dados de peso e estatura de crianças e gestantes das famílias acompanhadas. Estes nem sempre foram campos de preenchimento obrigatório no sistema de informação do Programa Bolsa Família. Esta é uma orientação que os gestores da rede municipal envolvidos com o Programa Bolsa Família conhecem, mas não é encontrada nos documentos orientadores do Governo Federal, pois eram feitas em comunicação direta aos gestores.

No Município do Rio de Janeiro existe uma plataforma digital de informação em saúde da APS, denominada plataforma SUBPAV (Subsecretaria de Promoção, Atenção Primária e Vigilância em Saúde). Esta agrega os dados advindos do e-SUS e outros sistemas de informação, como o sistema "próprio" do Programa Bolsa Família, desenvolvido pela gestão municipal. Nele, os profissionais digitam os dados das condicionalidades de saúde e são gerados relatórios de monitoramento das famílias acompanhadas. Estes dados migram para o sistema federal. Entretanto, este sistema "próprio" do Programa Bolsa Família possui limitações para a vigilância alimentar e nutricional. Os profissionais reclamam que o sistema é "engessado", não atendendo às necessidades do serviço. Uma proposta que surgiu em um GF é que o sistema pudesse gerar um alerta para identificar as pessoas em risco nutricional, o que seria útil para a ação de vigilância.

"Tinha que ter um sistema que alertasse, que desse o diagnóstico, nem que fosse o agente comunitário mesmo que pegasse peso e altura. Aí pronto, já fica vermelho aqui e na mesma hora agenda o paciente".

O diagnóstico nutricional coletivo também não é realizado como rotina. Independente dos profissionais inserirem os dados de peso e estatura para todas as idades, o sistema fornece a classificação do estado nutricional apenas para os menores de dois anos. Mesmo para estes dados, os profissionais não sabem como gerar um relatório capaz de ajudar na análise de um diagnóstico coletivo.

"A Plataforma SUBPAV não dá diagnóstico nutricional. Ela só dá peso, altura e a carteira [atualização vacinal]. Não dá nem o percentil. Tem que calcular um por um, e depois que coloca o IMC [índice de massa corporal] um por um. A gente tem que classificar tudo".

Poucas iniciativas locais visando a vigilância alimentar e nutricional das pessoas acompanhadas pelo Programa Bolsa Família foram citadas, ocorrendo principalmente em regiões de saúde que tinham nutricionistas atuando no NASF, as quais realizavam o diagnóstico nutricional coletivo de forma manual, usando planilhas do programa Excel (https://products.office.com/), o que inviabilizou a continuidade desta ação pela demanda de tempo para a atividade.

A falta de retorno da informação para as unidades, pelas instâncias de gestão, também não favorece o planejamento e a avaliação de estratégias de intervenção. Na maior parte dos relatos, o acompa- 
nhamento do crescimento das crianças e a vigilância alimentar e nutricional das famílias do Programa Bolsa Família não estavam estruturados.

"Não tem uma informação coletiva de estado nutricional. O certo seria aparecer ali o peso, a altura, o IMC e o próprio estado nutricional. Tinha uma planilha onde já tinha uma fórmula, que já calculava e dava o estado nutricional. Separava por risco para sobrepeso, obesidade, baixo peso e ai trabalhava com as equipes por grupos de risco. Acabou que a planilha morreu".

Estudo também realizado no Município do Rio de Janeiro apresenta a percepção de gestores da Saúde de que o acompanhamento das condicionalidades do Programa Bolsa Família impactou negativamente na continuidade do Sistema de Vigilância Alimentar e Nutricional (SISVAN) na rede municipal 20. No contexto do Município do Rio de Janeiro, este achado reflete a descontinuidade do monitoramento do estado nutricional, que, anteriormente, fazia parte dos critérios de inclusão de alguns programas de intervenção no setor Saúde (voltados ao cuidado de crianças e gestantes desnutridas). A partir do Programa Bolsa Família, que estabelece como critério de seleção a renda e não mais o estado nutricional, este monitoramento passa a não ser mais prioridade na agenda da gestão municipal de saúde.

Os dados referentes ao acompanhamento do estado nutricional do Programa Bolsa Família disponibilizados pelos sites oficiais são desatualizados, dificultando o acesso a esta informação pelos municípios, profissionais e até para estudos sobre este tema 21. Contudo, a principal fonte de dados do SISVAN é o Programa Bolsa Família, que contribuiu em 2013 com 85,78\% dos dados desse sistema 22.

A vigilância alimentar e nutricional, junto à prevenção e o cuidado das pessoas com agravos nutricionais e doenças relacionadas à alimentação, são ações que concretizam o compromisso do setor com a garantia do direito humano à saúde e do direito humano à alimentação adequada (DHAA), sendo, portanto, parte do papel do setor saúde na agenda da Política Nacional de Segurança Alimentar e Nutricional (PNSAN) 23.

A vigilância alimentar e nutricional é capaz de orientar as ações voltadas ao cuidado nutricional, em âmbito individual e familiar, pois articula as demais ações de alimentação e nutrição na saúde quando identifica pessoas em situação de risco nutricional que precisam de cuidado diferenciado 1,24.

\section{Estado nutricional das pessoas acompanhadas}

As narrativas dos profissionais de saúde quanto à situação alimentar e nutricional das pessoas acompanhadas pelo Programa Bolsa Família demonstraram desconhecimento e baixa valorização da vigilância alimentar e nutricional como instrumento de planejamento e gestão, e mesmo do diagnóstico nutricional como componente do cuidado em saúde.

Não é realizada nenhuma análise ou intervenção com base nos dados de peso e estatura coletados para o Programa Bolsa Família. Assim, a equipe não conhece o perfil nutricional das famílias.

"Quanto ao perfil nutricional, o médico não tem como saber, porque geralmente quem faz essa parte de peso e medida não é o médico. E a gente também não sabe quem é Bolsa Família ou não. Como é tanta gente, geralmente é até o próprio agente comunitário que pesa e mede, e entrega para a gente para lançar na plataforma SUBPAV. Os casos que não vieram na consulta, a gente não olha".

O conhecimento que os profissionais têm sobre o perfil nutricional vem das estratégias locais, em momentos pontuais de monitoramento ou da observação nas consultas individuais. Apesar da inexistência de dados consolidados deste perfil, os profissionais referem perceber grande prevalência de obesidade entre as crianças menores de cinco anos acompanhadas. Ainda que seja possível identificar o problema da obesidade, este não se desdobra em ação, seja pelos profissionais apresentarem dificuldades de intervenção ou por não existir um fluxo de cuidado organizado.

A desnutrição aparece em menor expressão, geralmente como causa secundária. Em que pese os avanços no controle da desnutrição infantil nas últimas décadas, este agravo ainda pode ser encontrado em grupos de extrema vulnerabilidade 25 .

A percepção dos profissionais é compatível com o perfil nutricional deste grupo em outro estudo em que as prevalências de baixo peso e obesidade foram maiores em municípios com maior desigualdade de renda 26.

As consequências da insegurança alimentar e nutricional na população recaem sobre o setor saúde 23 . Na APS, as ações de nutrição compõem a tradicional agenda da saúde pública, com foco no 
controle da desnutrição e de carências de micronutrientes. Mais recentemente, com os processos de transição epidemiológica e nutricional, as ações voltadas ao enfrentamento da epidemia de obesidade e das doenças crônicas não transmissíveis têm ganhado destaque na agenda, sem, no entanto, ter sido resolvida a questão anterior. Esta dupla carga sobre o setor saúde, somada à complexidade das questões para a garantia da segurança alimentar e nutricional, vem impondo importantes desafios para a organização da atenção nutricional 27.

O que se viu é que individualmente não existe um fluxo definido para o cuidado da obesidade, e coletivamente, não se gera diagnóstico nutricional. Deste modo, a magnitude do problema não se expressa e não são propostas intervenções.

\section{Oportunidade para o cuidado}

Apesar da visão mais burocrática do "pesar e medir" ter força nas narrativas, houve contrapontos, que expressam diferentes sentidos e potencialidades do acompanhamento realizado. Algumas narrativas ponderam que tal prática pode ser utilizada de forma estratégica, aproveitando este momento para a realização de visitas domiciliares, que proporcionam a criação de vínculos, melhor compreensão das condições de vida das famílias e maior tempo para avaliação clínica. Além disso, a ida à unidade de saúde para "pesar e medir" pode oportunizar um cuidado ampliado de saúde. Contudo, a melhor estratégia é que essa ação seja estruturada de forma integrada às linhas de cuidado.

"O pesar e medir se constitui um eixo de cuidado, principalmente para as crianças. (...) Até a prática do pesar e medir pode ser usada de forma estratégica. Aproveitar esse momento (...) para ir às casas, conversar, perguntar, se atualizar, fazer uma avaliação clínica. Tem enfermeiras que fazem isso. Ou se é um grupo para pesar e medir, pode organizar e aproveitar para fazer o acompanhamento nutricional, para ver essas crianças, ver uma questão (...) de saúde".

O monitoramento do crescimento da criança, utilizando os gráficos dos índices antropométricos do cartão de saúde da criança, nem sempre é realizado neste desenho de acompanhamento, mas para alguns profissionais, esta é uma oportunidade para o cuidado da saúde da criança.

"Então a criança que recebe o benefício está vinculada a este cuidado de seis em seis meses, que é pesar e medir para saber como está o seu desenvolvimento. É um indicador de nutrição, de desenvolvimento, de período sem doença. Você consegue ver se uma criança está bem, cresce bem, engorda, ganha o peso direitinho (...) então, assim, o significado do cuidado".

Entretanto, a baixa adesão das famílias é identificada pelos profissionais como obstáculo para melhores resultados. Os motivos dessa baixa adesão são controversos e precisam ser melhor compreendidos. As narrativas apontam para a dificuldade de compreensão, tanto por profissionais quanto pelas famílias, do propósito da realização das medidas antropométricas como parte do acompanhamento das condicionalidades. A não comunicação do diagnóstico e a inexistência de intervenção diante deste reforça o sentido burocrático para as famílias.

Embora o fluxo paralelo desta ação, não integrado às linhas de cuidado, seja o mais presente nas unidades que participaram dos GF, algumas reorientaram a ação do Programa Bolsa Família, integrando-a à rotina, ao longo dos anos de implementação deste acompanhamento.

"Quando começa a instaurar as consultas para este acompanhamento, inicia-se outra relação: 'E aí, mas você está com algum problema? E aí, como é que vamos? Está bem na escola? Tem algum problema em casa?' Na primeira vez é assim. Na segunda, é quando você vai construindo um vínculo, é quando você começa a entender e aquela família começa a se expor, porque, teoricamente, elas trouxeram a cultura do peso e da altura".

Quando os usuários percebem esta mudança transformam a sua expectativa em relação à ação. As unidades que conseguiram reorientar o modo de acompanhamento das condicionalidades do Programa Bolsa Família, o fizeram na direção de elementos que orientam o cuidado à saúde na APS, como a abertura para o diálogo, a construção de vínculo e a responsabilização, a fim de abarcar a dimensão cuidadora da saúde centrada no sujeito 16,28 .

A proposta da vigilância alimentar e nutricional como uma das ações de acompanhamento das condicionalidades de saúde, integrada às linhas de cuidado de saúde da criança e da gestante, permite o acompanhamento individual do estado nutricional destes grupos, identificando oportunamente situações de risco que demandam cuidado diferenciado. Coletivamente, reforça a análise da situação nutricional e a identificação no território de grupos mais vulneráveis à insegurança alimentar e nutri- 
cional e de seus determinantes, favorecendo a organização do cuidado aos agravos nutricionais. Para isso, a avaliação nutricional deve ser um procedimento realizado durante todas as consultas de acompanhamento desses grupos. Além disso, o aumento da obesidade entre adolescentes e adultos indica a necessidade de ampliar a vigilância alimentar e nutricional das famílias atendidas pelo Programa Bolsa Família também para estes grupos 22.

Priorizar ações voltadas à segurança alimentar e nutricional de grupos vulneráveis é ainda mais necessário frente ao quadro de recrudescimento da fome no país, diante do cenário de crise econômica e de uma política de austeridade fiscal, aliada ao desmonte das políticas sociais 29 . Este contexto se complexifica com a pandemia de COVID-19, que amplificou desigualdades existentes, comprometendo ainda mais o DHAA.

\section{Considerações finais}

Este estudo permitiu identificar que prevalece uma visão reducionista dessas condicionalidades de saúde do Programa Bolsa Família, apenas para garantir que a família não tenha o benefício cortado e para alcançar o cumprimento das metas de gestão. Ao longo das três etapas da pesquisa, os profissionais construíram uma reflexão crítica desta prática burocratizada e reconheceram sua potencialidade para o cuidado em saúde e nutrição das famílias.

A alta rotatividade de profissionais, equipes incompletas e o grande número de beneficiários atendidos por equipe tornam a realização das ações previstas para o acompanhamento das condicionalidades de saúde do Programa Bolsa Família um desafio. Entretanto, é preciso atentar que as condicionalidades não devem visar a meta por si só, mas a oportunidade de acesso ao cuidado integral de famílias vulneráveis, diante do fenômeno de "inversão do cuidado", em que sabidamente quem mais procura a unidade é quem menos precisa.

É necessário rever como as unidades de saúde se organizam para o diagnóstico e monitoramento do estado nutricional das famílias do Programa Bolsa Família, face a sinergia desta ação com a agenda do setor Saúde na segurança alimentar e nutricional e seu papel em subsidiar e avaliar ações individuais e coletivas voltadas à melhoria das condições de alimentação, nutrição e saúde da população. 


\section{Colaboradores}

J. J. Damião e E. Lobato contribuíram na concepção e delineamento do estudo, coleta e análise dos dados, redação e revisão crítica do manuscrito. J. P. Silva contribuiu na concepção e delineamento do estudo, análise dos dados e revisão crítica do manuscrito. C. V. C. Silva contribuiu na concepção e delineamento do estudo, coleta e análise dos dados e revisão crítica do manuscrito. L. M. C. Castro contribuiu na coleta e análise dos dados e revisão crítica do manuscrito. L. A. Maldonado contribuiu na análise dos dados e revisão crítica do manuscrito. A. A. Ribeiro contribuiu na concepção do estudo, análise dos dados e revisão crítica do manuscrito. Todos os autores aprovaram a versão final do artigo para publicação

\section{Informações adicionais}

ORCID: Jorginete de Jesus Damião (0000-00016591-3474); Evelyne Lobato (0000-0001-71010837); Juliana Paulo e Silva (0000-0003-2577-2341); Claudia Valéria Cardim da Silva (0000-0003-49395106); Luciana Maria Cerqueira Castro (00000003-2793-9950); Luciana Azevedo Maldonado (0000-0001-9718-7526); Alexandro Alves Ribeiro (0000-0002-6531-1753).

\section{Agradecimentos}

Os autores agradecem o financiamento do Conselho Nacional de Desenvolvimento Científico e Tecnológico (CNPq), por meio de chamada CNPq/ MS/SCTIE/DECIT/SAS/DAB/CGAN no 13/2017 - Pesquisas em Alimentação e Nutrição; à gestão central e territorial do Programa Bolsa Família do Município do Rio de Janeiro, aos gestores das unidades de saúde participantes e principalmente aos profissionais que estiveram conosco em todas as etapas do trabalho de campo.

\section{Referências}

1. Departamento de Atenção Básica, Secretaria de Atenção à Saúde, Ministério da Saúde. Marco de referência da vigilância alimentar e nutricional na atenção básica. Brasília: Ministério da Saúde; 2015.

2. Cotta RMM, Machado JC. Programa Bolsa Família e segurança alimentar e nutricional no Brasil: revisão crítica da literatura. Rev Panam Salud Pública 2013; 33:54-60.

3. Neves JA, Vasconcelos FAG, Machado ML, Recine E, Garcia GS, Medeiros MAT. The Brazilian cash transfer program (Bolsa Família): a tool for reducing inequalities and achieving social rights in Brazil. Glob Public Health 2020; 30;1-17.

4. Burlandy L. Transferência condicionada de renda e segurança alimentar e nutricional. Ciênc Saúde Colet 2007; 12:1441-51.

5. Carmo AS, Almeida LM, Oliveira DR, Santos LC. Influence of the Bolsa Família program on nutritional status and food frequency of schoolchildren. J Pediatr (Rio J.) 2016; 92:3817.

6. Moraes VD, Machado CV. O Programa Bolsa Família e as condicionalidades de saúde: desafios da coordenação intergovernamental e intersetorial. Saúde Debate 2017; 41(spe3): 129-43.

7. Ministério da Saúde, Secretaria de Atenção à Saúde, Departamento de Atenção Básica. Política Nacional de Alimentação e Nutrição. Brasília: Ministério da Saúde; 2013.

8. Damião Trevisani JJ. Avaliação da implementação das condicionalidades de saúde do Programa Bolsa Família e seu papel no cuidado à saúde - estudo de caso do município do Rio de Janeiro [Tese de Doutorado]. São Paulo: Faculdade de Saúde Pública, Universidade de São Paulo; 2012.

9. Onocko Campos R, Furtado JP, Passos E, Benevides R. Pesquisa avaliativa em saúde mental: desenho participativo e efeitos da narratividade. São Paulo: Hucitec Editora; 2013.

10. Ricoeur P. Tempo e narrativa. Campinas: Papirus; 1997.

11. Onocko Campos R. Fale com eles! O trabalho interpretativo e a produção de consenso na pesquisa qualitativa em saúde: Inovações a partir de desenhos participativos. Physis (Rio J.) 2011; 21:1269-86.

12. Ritchie J, Lewis J. Qualitative research practice: a guide for social science students and researchers. London: Sage Publications; 2003.

13. Ministério do Desenvolvimento Social e Combate à Fome. Portaria no 551, de 9 de novembro de 2005. Regulamenta a gestão das condicionalidades do Programa Bolsa Família. Diário Oficial da União 2005; 11 nov.

14. Carvalho AT, Almeida ER, Jaime PC. Condicionalidades em saúde do programa Bolsa Família - Brasil: uma análise a partir de profissionais da saúde. Saúde Soc 2014; 23:1370-82. 
15. Furtado SA. Programa Bolsa Família: percepção dos profissionais e beneficiários quanto ao acompanhamento das condicionalidades em saúde [Dissertação de Mestrado]. Lavras: Programa de Pós-graduação em Nutrição e Saúde, Universidade Federal de Lavras; 2019.

16. Cecilio LCO. As necessidades de saúde como conceito estruturante na luta pela integralidade e equidade na atenção em saúde. In: Pinheiro R, Mattos RA, organizadores. Os sentidos da integralidade na atenção e no cuidado à saúde. Rio de Janeiro: Centro de Estudos e Pesquisa em Saúde Coletiva, Instituto de Medicina Social, Universidade do Estado do Rio de Janeiro/Associação Brasileira de Saúde Coletiva; 2001. p. 113-26.

17. Giovanella L, Franco CM, Almeida PF. Política Nacional de Atenção Básica: para onde vamos? Ciênc Saúde Colet 2020; 25:1475-82.

18. Departamento de Atenção Básica, Secretaria de Atenção à Saúde, Ministério da Saúde. Manual de orientações sobre o Bolsa Família na saúde. 3a Ed. Brasília: Ministério da Saúde; 2010.

19. Brasil. Lei no 13.595 , de 5 de janeiro de 2018 . Altera a Lei no 11.350, de 5 de outubro de 2006, para dispor sobre a reformulação das atribuições, a jornada e as condições de trabalho, o grau de formação profissional, os cursos de formação técnica e continuada e a indenização de transporte dos profissionais Agentes Comunitários de Saúde e Agentes de Combate às Endemias. Diário Oficial da União 2018; 18 abr.

20. Ferreira MN. Programas de Transferência Condicionada de Renda e acesso aos serviços de saúde: um estudo da experiência do Programa Bolsa Família em Manguinhos, RJ [Dissertação de Mestrado]. Rio de Janeiro: Escola Nacional de Saúde Pública Sergio Arouca, Fundação Oswaldo Cruz; 2009.

21. Wolf MR, Barros Filho AA. Estado nutricional dos beneficiários do Programa Bolsa Família no Brasil - uma revisão sistemática. Ciênc Saúde Colet 2014; 19:1331-8.

22. Nascimento FA, Silva SA, Jaime PC. Cobertura da avaliação do estado nutricional no Sistema de Vigilância Alimentar e Nutricional brasileiro: 2008 a 2013. Rev Bras Epidemiol 2019; 22:e190028.
23. Alves KPS, Jaime PC. A política nacional de alimentação e nutrição e seu diálogo com a política nacional de segurança alimentar e nutricional. Ciênc Saúde Colet 2014; 19:4331-40.

24. Vitorino SAS. Avaliação da implantação da Vigilância Alimentar e Nutricional na Atenção Primária em Saúde: estudos de caso em municípios de pequeno porte de Minas Gerais [Tese de Doutorado]. Rio de Janeiro: Escola Nacional de Saúde Pública Sergio Arouca, Fundação Oswaldo Cruz; 2017.

25. Cardoso LO, Monteiro CA. Situação nutricional da população brasileira - cenário atual e futuros desafios. In: Ação Brasileira pela Nutrição e Direitos Humanos; Ministério da Saúde, organizadores. Textos de opinião: temas estratégicos para a Política Nacional de Alimentação e Nutrição (PNAN). Brasília: Organização Pan-Americana da Saúde; 2011. p. 89-118.

26. Silva DAS, Nunes HEG. Prevalência de baixo peso, sobrepeso e obesidade em crianças pobres do Mato Grosso do Sul. Rev Bras Epidemiol 2015; 18:466-75.

27. Associação Brasileira de Saúde Coletiva. Fortalecimento da agenda de segurança alimentar e nutricional na saúde - subsídios para as Conferências de Saúde e de Segurança Alimentar e Nutricional. Grupo Temático Alimentação e Nutrição em Saúde da Associação Brasileira de Saúde Coletiva. https://www.abrasco.org.br/ site/gtalimentacaoenutricaoemsaudecoletiva/ documentos/ (acessado em 23/Ago/2017).

28. Franco TB, Magalhães Junior HM. Integralidade na assistência à saúde: a organização das linhas do cuidado. In: Merhy EE, Magalhães Júnior HM, Rimoli J, Franco TB, Bueno WS, organizadores. O trabalho em saúde: olhando e experienciando o SUS. São Paulo: Hucitec Editora; 2003. p. 125-34.

29. Castro IRR. A extinção do Conselho Nacional de Segurança Alimentar e Nutricional e a agenda de alimentação e nutrição. Cad Saúde Pública 2019; 35:e00009919. 
Abstract

The study aimed to understand the narratives of primary healthcare workers in the city of Rio de Janeiro, Brazil, on the challenges and potentialities of food and nutritional surveillance activities in the context of the Brazilian Income Transfer Program, vis-à-vis the limits of its implementation. The study adopted a qualitative, evaluative, and participatory approach. From 2018 to 2019, five focus groups were held with 60 universitylevel healthcare workers from 24 health units and one focus group with 13 community health agents. The material transcribed from the focus groups was used to construct narratives for each group, producing a synthesis of the core arguments in the discourses. Five hermeneutic groups were later held for validation of the narratives and in-depth discussions. The last meeting identified problems and proposals related to follow-up of the Brazilian Income Transfer Program. In practice, followup of the conditionalities is limited to "weighing and measuring" individuals without necessarily conducting a nutritional diagnosis to identify cases of nutritional risk and intervention. Factors contributing to this scenario include practices such as mass assessment ("mutirão") at the end of coverage by Brazilian Income Transfer Program and anthropometric measurements taken by community health agents, with no link to the existing lines of care. A bureaucratic vision of these health conditionalities prevails, merely to guarantee that the family does not lose the benefit and that the program's administrative targets are met. Over the course of the study, the healthcare workers developed a critical analysis of this practice and acknowledge their own potential to care for the families' health and nutrition.

Primary Health Care; Nutrition Policy; Nutritional Surveillance; Food Security

\section{Resumen}

Se procuró comprender los relatos de los profesionales de atención primaria en salud del municipio de Río de Janeiro, Brasil, sobre los desafíos y las potencialidades de las acciones de vigilancia alimentaria y nutricional, en el contexto del Programa Bolsa Familia, frente a los límites de su implementación. Se trata de una investigación con un abordaje cualitativo, evaluativo y participativo. Se formaron, entre 2018 y 2019, cinco grupos focales $(G F)$ con 60 profesionales de nivel superior de 24 unidades de salud y un GF con 13 agentes comunitarios de salud. En base al material transcrito de los GF, se construyeron relatos para cada grupo, produciendo una síntesis de los núcleos argumentales presentes en las conversaciones. Posteriormente, se compusieron cinco grupos hermenéuticos para la validación de los relatos y profundización de las discusiones. En el último encuentro se identificaron los problemas y propuestas relacionadas con el seguimiento del Programa Bolsa Familia. En la práctica, el seguimiento de las condicionalidades está reducido a "pesar y medir" a personas, sin que necesariamente se realice un diagnóstico nutricional para la identificación de casos de riesgo e intervención. Prácticas como el "mutirão" (actividad colectiva voluntaria), al final de la vigencia del Programa Bolsa Familia, y la realización de medidas antropométricas por parte de los agentes comunitarios de salud, sin vinculación con la agenda de las líneas de cuidado existentes, contribuyen a este escenario. Prevalece una visión burocrática de esas condicionalidades de salud, solamente para garantizar que a la familia no se le suprima el beneficio y el cumplimiento de las metas de gestión del Programa Bolsa Familia. A lo largo de la investigación, los profesionales construyeron una reflexión crítica de esta práctica $y$ reconocieron su potencialidad para el cuidado en salud y nutrición de las familias.

Atención Primaria de Salud; Política Nutricional; Vigilancia Nutricional; Seguridad Alimentaria
Recebido em 20/Ago/2020

Versão final reapresentada em 12/Mar/2021

Aprovado em 19/Mar/2021 